\title{
Primary hyperoxaluria type III - a model for studying perturbations in glyoxylate metabolism
}

\section{Ruth Belostotsky ${ }^{1}$, James Jonathon Pitt ${ }^{2}$ and Yaacov Frishberg ${ }^{1,3}$}

${ }^{1}$ Division of Pediatric Nephrology, Shaare Zedek Medical Center, Jerusalem, Israel.

${ }^{2}$ VCGS Pathology, Murdoch Childrens Research Institute, Royal Children's Hospital, Parkville, Victoria. Australia.

${ }^{3}$ Hadassah-Hebrew University School of Medicine, Jerusalem, Israel.

Abstract word counter: 224

Text word count: 3622

\section{Corresponding Author:}

Ruth Belostotsky, Division of Pediatric Nephrology, Shaare Zedek Medical

Center, P.O.Box 3235, Jerusalem 91031, Israel; Tel: 972-2-6666749; Fax: 972-2-

6555484; e-mail: ruthb@ekmd.huji.ac.il 


\section{Abstract}

Perturbations in glyoxylate metabolism lead to the accumulation of oxalate and give rise to primary hyperoxalurias, recessive disorders characterized by kidney stone disease. Loss-offunction mutations in HOGAl (formerly DHDPSL) are responsible for primary hyperoxaluria type III. HOGA1 is a mitochondrial 4-hydroxy-2-oxoglutarate aldolase catalyzing the fourth step in the hydroxyproline pathway. We investigated hydroxyproline metabolites in the urine of patients with primary hyperoxaluria type III using gas chromatography - mass spectroscopy. Significant increases in concentrations of 4-hydroxy2-oxoglutarate and its precursor and derivative 4-hydroxyglutamate and 2,4dihydroxyglutarate, respectively, were found in all patients as compared to carriers of the corresponding mutations or healthy controls. Despite a functional block in the conversion of hydroxyproline to glyoxylate - the immediate precursor of oxalate - the production of oxalate increases. To explain this apparent contradiction we propose a model of glyoxylate compartmentalization in which cellular glyoxylate is normally prevented from contact with the cytosol where it can be oxidized to oxalate. We propose that HOGA1 deficiency results in the accumulation of 4-hydroxy-2-oxoglutarate in the mitochondria and its transport into the cytosol where it is converted to glyoxylate by a different cytosolic aldolase. In human hepatocyte cell lines we detected a cytosolic 4-hydroxy-2-oxoglutarate aldolase activity not due to HOGA1. These studies provide a diagnostic tool for primary hyperoxaluria type III and shed light on glyoxylate metabolism and the pathogenesis of primary hyperoxalurias.

Key words: HOGA1; PHIII; hydroxyproline pathway; glyoxylate; oxalate; DHDPSL. 


\section{Introduction}

Our recent identification of a previously unknown gene encoding 4-hydroxy-2-oxoglutarate aldolase (HOGA1), and demonstrating the role of mutations in the HOGA1 (formerly DHDPSL) gene in type III primary hyperoxaluria (PHIII) [1], created the need to elucidate the effect of deficiency of this enzyme on oxalate formation. Recent publications presented controversial speculations on this subject [2-4].

HOGA1 is the last enzyme in the hydroxyproline-glyoxylate pathway within the mitochondria: it catalyzes the formation of glyoxylate, the precursor of oxalate. Since primary hyperoxaluria is characterized by accumulation of calcium oxalate crystals, we initially proposed that in patients with PHIII, HOGAl bears activating mutations, leading to glyoxylate over-production. However, detection of additional mutations, including nonsense ones [2,4] and the position of mutated amino acids in the 3D structure of HOGA1 [3] suggested that these mutations are actually damaging. These observations suggest that loss of HOGA1 enzymatic activity causes rerouting of its substrate, R-4-hydroxy-2-oxoglutarate (HOG) to glyoxylate and subsequent conversion to oxalate.

Glyoxylate is produced by two pathways: the oxidation of glycolate contributed by vegetarian food, and catabolism of hydroxyproline supplied by turnover of internal collagen and protein from animal food (Fig. 1). These processes are restricted to peroxisomes and mitochondria, respectively. In humans, glyoxylate is metabolized to glycine by the peroxisomal alanine/glyoxylate aminotransferase (AGT). A minor portion of glyoxylate is oxidized to oxalate by the cytosolic lactate dehydrogenase (LDH). We propose that under normal conditions, glyoxylate is prevented from contact with cytosolic LDH. The delivery of mitochondrial glyoxylate, generated from hydroxyproline, to the peroxisome is accomplished by its conversion to glycolate by the mitochondrial glyoxylate reductase (GRHPR) and reciprocal oxidation to glyoxylate by peroxisomal glycolate oxidase (GO). 
Our hypothesis was that if HOGA1 is deficient, HOG accumulates and is transported from the mitochondria into the cytosol where it is converted to glyoxylate by a different cytosolic aldolase. This pathologic process interferes with glyoxylate compartmentalization.

\section{Materials and Methods}

\section{Patients}

Nine patients from 4 families of Ashkenazi-Jewish descent were studied. The diagnosis was established by the detection of bi-allelic mutations in HOGAl. They all had persistent hyperoxaluria (oxalate excretion of $1.2 \pm 0.5 \mathrm{mmol} / 1.73 \mathrm{~m}^{2} / \mathrm{d}$ [range $0.54-2.24$; normal values $<0.49]$ ). Six patients formed calcium-oxalate kidney stones. Two additional groups were similarly evaluated: 8 parents (obligatory carriers) and healthy controls. All samples from patients and their relatives were obtained after receiving informed consent and approval by the SZMC Helsinki Committee (number 1/09).

\section{GC-MS analysis of urine and cellular fractions}

Preparation of standards is described in Supplementary Methods. Two hundred microlitres of urine or reaction mixtures was mixed with $20 \mu \mathrm{L}$ of $1 \mathrm{M}$ methoxyamine hydrochloride adjusted to $\mathrm{pH} 9.4$ with $25 \%$ ammonia and containing internal standards $(1.0 \mathrm{mM}$ each of 3,3-dimethylglutaric acid, ${ }^{2} \mathrm{H}_{2}$-glycolic acid and ${ }^{2} \mathrm{H}_{3}$-glyceric acid). The mixture was incubated at $65^{\circ} \mathrm{C}$ for 60 minutes, cooled, acidified with $10 \mu \mathrm{L} 6 \mathrm{M}$ hydrochloric acid, saturated with solid sodium chloride and extracted with $1 \mathrm{~mL}$ of ethyl acetate:propan-2-ol (5:1 v:v). The organic phase was decanted, dried under an air stream and trimethylsilyl derivatives were prepared by incubation for 30 minutes at $80^{\circ} \mathrm{C}$ with a mixture of $80 \mu \mathrm{L}$ of N,O-bis(trimethylsilyl)trifluoroacetamide containing $1 \%$ trimethylchlorosilane and $20 \mu \mathrm{L}$ pyridine. $100 \mu \mathrm{L}$ of iso-octane was added and the mixture was analyzed by gas chromatography-mass spectrometry (Agilent 5973 system). An HP-5MS column was used 
(30m x $0.25 \mathrm{~mm}$ internal diameter, $0.25 \mu \mathrm{m}$ film thickness). One microlitre of sample was injected in split-less mode, the column was held at $80^{\circ} \mathrm{C}$ for 1 minute then ramped to $300^{\circ} \mathrm{C}$ at $8^{\circ} \mathrm{C}$ per minute. The mass spectrometer was operated in either scanning mode (50 to 550 $\mathrm{m} / \mathrm{z}$ at 2.4 scans/second) or in selected ion monitoring mode using the ions shown in Supplemental Table. Chiral analysis of HOG and LC-MSMS analysis of 4-hydroxyglutamic acid and hydroxyproline are described in Supplementary Methods.

\section{Isolation of mitochondria}

All procedures were performed at $4^{\circ} \mathrm{C}$ : confluent SK-HEP-1 (ATCC\#HTB-52) cells were washed with PBS, resuspended in mitochondrial isolation buffer (MIB: $10 \mathrm{mM}$ HEPES$\mathrm{KOH}, \mathrm{pH}$ 7.4, $200 \mathrm{mM}$ mannitol, $70 \mathrm{mM}$ sucrose, and protease inhibitor cocktail). Cells were homogenized in a glass homogenizer using a tight pestle. Unbroken cells, nuclei, and large debris were pelleted at $600 \mathrm{~g}$ for 10 minutes following by centrifugation of supernatant at $10000 \times g$ for $15 \mathrm{~min}$. The cytosolic supernatant was separated. The pellet was washed and resuspended in MIB following three rounds of sonication for 10 seconds. The mitochondrial fraction was centrifuged at $10000 \times g$ for $15 \mathrm{~min}$ and the pellet was discarded. The fractions were kept at $-70{ }^{\circ} \mathrm{C}$.

\section{Measurements of HOG decomposition}

The decrease of HOG concentrations following incubation with cytosolic and mitochondrial fractions of SK-HEP-1 cells was measured using GC-MS analyses. The reaction mixtures contained cell fractions diluted to $2.5 \mathrm{mg} / \mathrm{ml}$ protein in MIB and $50 \mu \mathrm{M}$ of HOG. Cell fractions were also deproteinized by ultrafiltration through Vivaspin columns with molecular weight cutoff $5 \mathrm{kDa}$ (Sartorius, Hannover, Germany) to control for possible non-enzymatic HOG conversion. The dilution was in the same proportion as for the non-filtered fractions. Samples were incubated for 4 hours at $37^{\circ} \mathrm{C}$ and frozen until measurement. 
Catalysis of HOG decomposition (Fig. 2; stage 2) by the cytosolic fraction was also detected in situ by measuring the production of glyoxylate and pyruvate in a coupled reaction with $\mathrm{LDH}$ and NADH. The reaction mixture contained cytosolic fraction diluted to $0.1 \mathrm{mg} / \mathrm{ml}$ protein, $6 \mathrm{U}$ LDH type XI from rabbit muscle (Sigma) and $200 \mu \mathrm{M}$ HOG in MIB. The reaction was initiated by the addition of NADH (to a $150 \mu \mathrm{M}$ concentration) and followed by measuring a decrease in $A_{340}$ at room temperature. The decrease of $A_{340}$ in a reaction mixture without external HOG served as a control curve.

\section{Western blot}

Concentrated cytosolic and mitochondrial fractions of SK-HEP-1 cells containing equal amounts of protein $(50 \mathrm{mg} / \mathrm{ml})$ were separated by SDS-polyacrylamide gel electrophoresis, transferred to PVDF membrane (Pall Corporation, FL) and immunoblotted using 1/100 diluted polyclonal anti-HOGA1 antibodies (Santa Cruz Biotechnology) and 1/10,000 diluted rabbit anti-goat HRP conjugated antibody (Jackson ImmunoResearch Laboratories, Inc.). Detection was performed using EZ-ECL chemiluminescence kit (Biological Industries, Israel). As a positive control we used recombinant affinity-purified HOGA1 protein [1].

\section{Results}

\section{Detection of HOG and its derivatives in urine of patients with PHIII}

We investigated whether HOG accumulates and is transported into the cytosol under PHIII conditions. Since liver biopsies from PHIII patients were unavailable to us, we investigated the urinary excretion of HOG and its derivatives. We hypothesized that there may be at least three alterative metabolic routes for HOG metabolism: accumulation of 4hydroxyglutamate, the metabolite immediately upstream of HOG; the reduction of HOG to 2,4-dihydroxyglutarate; and the cleavage of HOG to glyoxylate, resulting in the formation of glycolate and oxalate (Fig. 2; steps 1 and 2). 
Persistent hyperoxaluria was found in all affected individuals. Urinary concentrations of HOG, 2,4-dihydroxyglutarate and 4-hydroxyglutamate were measured by gas chromatography-mass spectroscopy (GC-MS) analyses in patients with PHIII, their parents and in controls. Routine organic acid GC-MS screening was unreliable for diagnosing PHIII because HOG and 2,4-dihydroxyglutaric were barely detectable (data not shown), presumably due to the poor extraction efficiency of these polar metabolites. We therefore developed a more sensitive method, by replacing ethyl acetate with a more polar extraction solvent (ethyl-acetate:propan-2-ol) and formation of methoxyamine derivatives to improve extraction efficiency. The resulting data are summarized in Fig. 3. There was a $\sim 50$ fold increase in the mean levels of HOG in PHIII patients compared to controls and almost complete discrimination between PHIII (1.7 - $118 \mu \mathrm{mol} / \mathrm{mmol}$ creatinine, $\mathrm{n}=9)$ and controls $(0.07-2.8, \mathrm{n}=16)$. Two chromatographic peaks of 2,4-dihydroxyglutarate are presented separatly: the meso-isomer (1) and the mixture of two optically active isomers (2) (see below). The analyses of all HOG derivatives provided evident discrimination between controls and PHIII patients. Levels of glycolate, glycerate (markers for PHI and PHII, respectively) and hydroxyproline were indistinguishable from controls (data not shown). These data confirm that in PHIII patients the accumulation of HOG results in its release into the cytosol and subsequent partial excretion in the urine, in combination with its related metabolites. The cytosolic HOG utilization via its decomposition to glyoxylate was consequently investigated in an in vitro system.

\section{The metabolism of HOG within the cytosol of human hepatocytes}

To check our assumption that the cytosol contains an alternative HOG-aldolase activity, we used human hepatocyte cell line SK-HEP-1. The first step was to investigate whether HOG is metabolized in both the cytosolic and the mitochondrial fractions. This was assessed by GC-MS assays of externally added HOG following incubation with either of these fractions, 
as well as with the fractions depleted of proteins by ultrafiltration (control). The amount of HOG decreased by about $99 \%$ in both mitochondrial and cytosolic fractions (Fig. 4). Similar results were obtained in Hep-G2 cells (data not shown).

It was not possible to measure glyoxylate production in order to confirm the cytosolic HOGaldolase activity. This was due to oxidation of glyoxylate to oxalate by LDH. Indeed, the addition of external glyoxylate to the cytosolic fraction resulted in its complete disappearance following 2- hour incubation.

Since a fixed-point assay was inapplicable, we implemented an alternative assay to evaluate the rate of in situ production of glyoxylate and pyruvate by measuring the decrease of $\mathrm{NADH}$ in a coupled reaction with recombinant LDH. The slope of the curve with added HOG is much steeper than that of the control curve (Fig. 4B). These results suggest the presence of cytosolic HOG-aldolase activity.

To confirm that cytosolic activity is not due to partial cytosolic localization of HOGA1, we performed immunobloting of mitochondrial and cytosolic fractions of SK-HEP-1 cells using anti-HOGA1 antibodies (Fig. 5). Our results confirm the exclusively mitochondrial localization of this protein.

GC-MS analyses reveal minor production of 2,4-dihydroxyglutarate in the cytosolic fraction (not shown), indicating that HOG reduction takes place within the cytosol.

\section{Analyses of isomeric forms of HOG, 2,4-dihydroxyglutarate and 4-hydroxyglutamate}

We used scanned spectra to confirm HOG, 2,4-dihydroxyglutarate and 4-hydroxyglutamate by spectral matching and co-elution with the synthesized standards ( Fig. S1). Chiral analysis showed that excreted HOG was almost entirely the R-isomer in PHIII patients (Fig. $\mathrm{S} 2,94-100 \%$ of total, $\mathrm{n}=12$ ) as expected from the known metabolic pathway. HOG was near the limit of detection in some of the non-PHIII subjects but R-HOG was also the major 
isomer in obligate heterozygotes $(71-100 \%, \mathrm{n}=5)$ and controls $(74-100 \%, \mathrm{n}=3)$ in whom it could be detected.

2,4-dihydroxyglutarate was present as two peaks in the GC-MS urine profiles (Fig. S3) with almost identical mass spectra in PHIII patients. 2,4-dihydroxyglutaric acid can exist as three possible isomers: a stereoisomeric pair $(2 \mathrm{R}, 4 \mathrm{R}$ and $2 \mathrm{~S}, 4 \mathrm{~S})$ and a meso-isomer $(2 \mathrm{~S}, 4 \mathrm{R})$. The 2S,4R meso-isomer has different physical properties to the stereoisomeric pair and separates (peak at 15.15 min, Fig. S3) from the first two isomers on the non-chiral column used. In contrast, the $2 \mathrm{R}, 4 \mathrm{R}$ and $2 \mathrm{~S}, 4 \mathrm{~S}$ stereo-isomers have identical properties and co-eluted as a single peak at 15.33 min. The absolute stereo configuration of this peak in PHIII patients was not determined but, given its origin from R-HOG, it is likely that it is mainly the $2 \mathrm{R}, 4 \mathrm{R}$ isomer. The finding of at least two 2,4-dihydroxyglutaric isomers suggests that more than one enzyme is involved in R-HOG reduction. The ratio of the optically active 2,4dihydroxyglutarate isomers peak to the meso-isomer peak was consistently high in PHIII patients $(2.8-25)$.

\section{Discussion}

In the mitochondrial hydroxyproline pathway, HOGA1 catalyzes the formation of glyoxylate and pyruvate from HOG. In order to explain the intriguing consequences of HOGA1 loss-of-function mutations leading to oxalate accumulation, we formed two postulates: 1. Sub-cellular glyoxylate localization is restricted to mitochondria and peroxisomes in normal cells. The flux across these compartments is provided by the reduction of glyoxylate to glycolate by mitochondrial GRHPR and reciprocal oxidation by peroxisomal GO. This mechanism prevents glyoxylate from oxidation by cytosolic LDH. 2 . If HOGA1 is deficient, HOG accumulates in the mitochondria and is transported into the cytosol where it is converted to glyoxylate by another cytosolic enzyme with HOG-aldolase 
activity. Cytosolic glyoxylate becomes available to LDH resulting in increased oxalate production.

\section{What are the theoretical consequences of cytosolic localization of glyoxylate?}

It is accepted that glyoxylate is distributed within the mitochondria, cytosol and peroxisomes [5-7]. Cytosolic glyoxylate can serve as a substrate of 3 reactions catalyzed by 2 cytosolic enzymes: 1. Reduction to glycolate by GRHPR, an enzyme that can use both NADH and NADPH as co-factors (Fig. 2; step 3); 2. Oxidation to oxalate by LDH in the presence of $\mathrm{NAD}^{+}$(Fig. 2; step 4); 3. Reduction to glycolate by LDH in the presence of NADH but not NADPH (Fig. 2; step 5).

When NADH is used as a cofactor, GRHPR and LDH have similar specificity constants to glyoxylate [8]. However, since cytosolic NADH/ $\mathrm{NAD}^{+}$ratio is very low (about $2 \times 10^{-3}$ ), both reactions are unfavorable. On the other hand, GRHPR, but not LDH, can use NADPH as a cofactor. The NADPH/ NADP ${ }^{+}$cytosolic ratio is about $10^{2}$ which directs the reduction of glyoxylate via this route.

The specificity constant of glyoxylate oxidation by LDH $\left(30-100 \mathrm{~s}^{-1} \mathrm{mM}^{-1}\right)$ is very similar to that of the reduction by GRHPR $\left(67 \mathrm{~s}^{-1} \mathrm{mM}^{-1}\right)$ [8]. The availability of either of the corresponding cofactors and relative cellular concentrations of LDH and GRHPR will determine the preferred reaction. In fact, both the NAD+/ NADPH ratio within the cytosol (10:1) and LDH/GRHPR transcription ratio (130:1) (http://smd.stanford.edu/cgi-

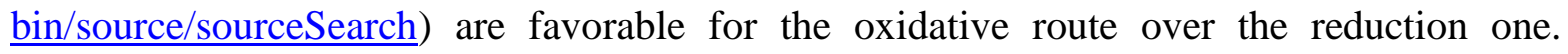
However, in reality only a minor portion of glyoxylate is converted to oxalate, while the majority follows the glycolate-glycine route. According to the calculations provided by Knight et. al. based on the comparison of oxalate excretion by individuals on diets poor and rich in hydroxyproline, only $0.5 \%$ of glyoxylate resulting from hydroxyproline is converted to oxalate [9]. This observation corroborates that the GRHPR route is significantly more 
favorable than LDH oxidation. This may be reached by preferable access of GRHPR to glyoxylate at the site of its production, the mitochondria.

Evaluation of relative activities of glyoxylate reduction versus oxidation can be calculated from experiments on oxalate/glycolate excretion by HepG2 cells, where the amount of excreted glycolate was 20 times higher than that of oxalate [5]. However, when radioactive glyoxylate was added to the medium, the excretion ratio between radioactive glycolate and oxalate was about 3:1. These observations confirm that once in the cytosol, glyoxylate is readily oxidized, and that this process hardly ever occurs under normal conditions.

\section{Glyoxylate and it precursors in the mitochondria}

HOGA1 deficiency should lead to accumulation of its substrate. Our data confirm that the level of HOG excreted in the urine by patients with PHIII is $~ 50$ times higher on average than that of the controls, implying that loss of HOGA1 activity results in transportation of the substrate into the cytosol.

Under normal conditions most of the HOG is decomposed to glyoxylate. We assume that glyoxylate is immediately reduced to glycolate by mitochondrial GRHPR. This enzyme was initially considered to be cytosolic, but later its mitochondrial localization was established in human hepatocytes [5] and in CHO cells transfected with human GRHPR cDNA [10]. The MitoProt II program predicts high probability of its export to the mitochondria (0.92).

The question whether $\mathrm{LDH}$ is expressed in the mitochondria of hepatocytes is intriguing. Out of four human $L D H$ genes only $L D H A$ and $L D H B$ are expressed in the liver with particular abundance of $L D H A$ [8]. Both are solely cytosolic proteins [11]. Computational analysis of all transcripts does not support mitochondrial export and localization of LDHA and $L D H B$ as well [12].

\section{The metabolism of HOG in PHIII conditions}


Our data demonstrate that loss-of-function mutations in HOGAl in patients with PHIII result in increased urinary excretion of HOG and its metabolites. This fact disproves the theory that glyoxylate is a substrate rather than a product of HOGA1 under physiological conditions [4]. As we postulated, HOGA1 deficiency uncovers cytosolic 4-hydroxy-2oxoglutarate aldolase activity. We assume that this activity represents a side action of a cytosolic pyruvate aldolase. Aldolases are known to be rather non-specific and to tolerate a wide range of aldehydes as acceptor compounds [13-14] Co-distribution of mitochondrial and cytosolic HOG aldolase activities was reported with regard to rat liver [15]. Kobes and Dekker [16] who purified an enzyme with HOGA activity from bovine liver observed two closely migrating protein bands, both with aldolase activity. We consider $\mathrm{N}$ acetylneuraminate lyase (NPL) to be the most probable aldolase which may provide cytosolic formation of glyoxylate from HOG. This cytosolic enzyme has a rather high homology to HOGA1, yet it is lacking the mitochondrial signal. However, other aldolases, such as fructose-bisphosphate aldolase, could be involved in this pathogenic process. Future investigation of cytosolic HOG pathway may provide a practical therapeutic approach for PHIII patients utilizing specific aldolase inhibitors.

Within the context of HOGA1 deficiency a portion of HOG is converted to 2,4dihydroxyglutarate resulting in dramatic increase of its level in the urine of PHIII patients. Reduction of accumulating keto-acids to the hydroxy-acids occurs in several inborn errors of metabolism e.g. reduction of phenylpyruvate in phenylketonuria [17]. Our preliminary experimental findings suggest that the reduction of HOG takes place within the cytosol. The resolution of at least two separate isomers of 2,4-dihydroxyglutarate suggests that more than one enzyme may be involved in this reduction and we cannot exclude their dual localization. We also demonstrated the accumulation of 4-hydroxyglutamate which is most likely due to 
build-up back along the hydroxyproline pathway caused by the reversibility of 4hydroxyglutamate aminotransferase [18-19].

We consider that in patients with PHIII, HOG and its precursor 4-hydroxyglutamate accumulate in the mitochondria and are released into the cytosol. Once in the cytosol, HOG and its product (2,4-dihydroxyglutarate) and precursor (4-hydroxyglutamate) are excreted in the urine and the residual HOG is converted into glyoxylate and pyruvate. Glyoxylate is metabolized in two routes: the oxidation with $\mathrm{LDH}$ and the reduction with cytosolic GRHPR.

\section{Compartmentalization theory and various hyperoxaluria conditions}

Compartment restriction of glyoxylate is not the only compartmentalization in this pathway, with the most prominent example being the localization of AGT. This enzyme provides the final step of glyoxylate removal. While human AGT is peroxisomal, it is distributed differently in various species in accordance with their animal food consumption. In carnivores, where the major source of oxalate is hydroxyproline, AGT is mitochondrial whereas in herbivores, where vegetable-derived glycolate intake is high, it is peroxisomal. In omnivores, it is both mitochondrial and peroxisomal [20-21]. This phenomenon underscores the importance of the proximity between glyoxylate production and utilization pathways.

The combination between the G170R mutation together with the P11L polymorphism in human AGT, erroneously targets the protein into the mitochondria and is responsible for about $30 \%$ of PHI conditions [22]. The G170R mutation has minor effect on specific activity (50-90\% of normal) and on affinity for PLP [23-24]. Thus, patients bearing this mutation have at least as high AGT activity as healthy individuals heterozygous for other AGT mutations. The statement that in a mitochondrial environment AGT is metabolically inefficient is doubtful since AGTs have dual localization in many species including some 
primates. It was also demonstrated that Pro11Leu polymorphism provides benefit in human populations with predominantly meat-eating ancestral diets [25].

As we alluded to earlier, mitochondrial AGT matches the location of glyoxylate, generated from hydroxyproline. Whereas most of the mitochondrial glyoxylate interacts with the mistargeted AGT, an oxalate formation may result from the metabolism of dietary glycolate. The content of glycolate is $9-13 \mathrm{mg} / \mathrm{day} / 2,500 \mathrm{kcal}$ in the high- and low-protein diets respectively [26]. This suggests that glycolate consumption cannot substantially impact on urinary oxalate excretion (normal daily range 15-45 mg). The amount of glyoxylate generated from the daily collagen turnover is about $170-260 \mathrm{mg}$ and it may double as a result of meat consumption. Thus, glycolate consumption contributes only $2-7 \%$ to the daily glyoxylate production whereas the majority is derived from hydroxyproline. The pathologic phenotype of PHI within the context of mistargeting may be explained by intramitochondrial competition between AGT and GRHPR for the substrate (Fig. 6). This novel view point of the metabolic consequences of AGT mistargeting may provide a paradoxical approach to the cure of this disease: inhibiting GRHPR activity will enable mitochondrial AGT to metabolize all the glyoxylate.

The compartmentalization theory sheds light on the pathogenesis underlying all 3 forms of primary hyperoxaluria as depicted in Fig. 6.

\section{Diagnostic implications}

Our metabolite findings have direct practical implications for improving the diagnosis of PHIII. We have developed a sensitive, fast and inexpensive method, capable of detecting HOG and its metabolites even in controls. This biochemical screening test provides a way to prioritize patients for subsequent genetic confirmation and allow more widespread testing to gain a better idea of the prevalence of PHIII. Of note, PHIII seems to have a milder phenotype compared with the other 2 types of $\mathrm{PH}$ and may overlap with the so called 
idiopathic calcium-oxalate stone disease. Interestingly, although some affected individuals form their first or recurrent kidney stone during the second or third decade, the most prevalent phenotype is of kidney stone disease manifesting in infancy or early childhood with a mild course later on in life. The exact mechanism underlying this observation remains elusive and is the subject of ongoing research.

Importantly, the novel testing was combined with analyses for glycolic and glyceric acids and this provides a single screening test for detecting and distinguishing between all three types of genetic hyperoxaluria.

In conclusion, theoretical analyses of the glyoxylate pathway and the consequences of mutations in $A G T, G R H P R$ and HOGAl led us to suggest the hypothesis of glyoxylate compartmentalization. Here we provide experimental confirmation of this hypothesis and considerations for the possible consequences of deficiencies in these enzymes. In particular, we investigated the paradoxical effect of HOGA1 deficiency on oxalate overproduction and present experimental explanations of HOG rerouting. The practical method of analysis of urinary excretion of HOG, 2,4-dihydroxyglutarate and 4-hydroxyglutamate can be introduced into clinical practice as a diagnostic tool.

\section{Acknowledgments}

The authors are indebted to Prof. Hanna Mandel for helpful advice. Sections of this work were supported by the Victorian Government's Operational Infrastructure Support Program.

\section{Conflict of interest}

The authors declare that no conflict of interest exists.

\section{References}


1. Belostotsky R, Seboun E, Idelson GH, Milliner DS, Becker-Cohen R, Rinat C, Monico CG, Feinstein S, Ben-Shalom E, Magen D, et al (2010) Mutations in DHDPSL are responsible for primary hyperoxaluria type III. Am J Hum Genet 87:392-399

2. Monico CG, Rossetti S, Belostotsky R, Cogal AG, Herges RM, Seide BM, Olson JB, Bergstrahl EJ, Williams HJ, Haley WE, et al (2011) Primary hyperoxaluria type III gene HOGA1 (formerly DHDPSL) as a possible risk factor for idiopathic calcium oxalate urolithiasis. Clin J Am Soc Nephrol 6:2289-2295

3. Riedel TJ, Johnson LC, Knight J, Hantgan RR, Holmes RP, Lowther WT (2011) Structural and biochemical studies of human 4-hydroxy-2-oxoglutarate aldolase: implications for hydroxyproline metabolism in primary hyperoxaluria. PLoS One 6:e26021

4. Williams EL, Bockenhauer D, Van't Hoff WG, Johri N, Laing C, Sinha MD, Unwin R, Viljoen A, Rumsby G (2012) The enzyme 4-hydroxy-2-oxoglutarate aldolase is deficient in primary hyperoxaluria type 3. Nephrol Dial Transplant. Epub ahead of print

5. Baker PR, Cramer SD, Kennedy M, Assimos DG, Holmes RP (2004) Glycolate and glyoxylate metabolism in HepG2 cells. Am J Physiol Cell Physiol 287:C1359-1365

6. Coulter-Mackie MB (2006) 4-Hydroxyproline metabolism and glyoxylate production: A target for substrate depletion in primary hyperoxaluria? Kidney Int 70:1891-1893

7. Dijcker JC, Plantinga EA, van Baal J, Hendriks WH (2011) Influence of nutrition on feline calcium oxalate urolithiasis with emphasis on endogenous oxalate synthesis. Nutr Res Rev 22:1-15

8. Mdluli K, Booth MP, Brady RL, Rumsby G (2005) A preliminary account of the properties of recombinant human Glyoxylate reductase (GRHPR), LDHA and LDHB 
with glyoxylate, and their potential roles in its metabolism. Biochim Biophys Acta $1753: 209-216$

9. Knight J, Jiang J, Assimos DG, Holmes RP (2006) Hydroxyproline ingestion and urinary oxalate and glycolate excretion. Kidney Int 70:1929-1934

10. Behnam JT, Williams EL, Brink S, Rumsby G, Danpure CJ (2006) Reconstruction of human hepatocyte glyoxylate metabolic pathways in stably transformed Chinese-hamster ovary cells. Biochem J 394:409-416

11. Yoshida Y, Holloway GP, Ljubicic V, Hatta H, Spriet LL, Hood DA, Bonen A (2007) Negligible direct lactate oxidation in subsarcolemmal and intermyofibrillar mitochondria obtained from red and white rat skeletal muscle. J Physiol 582:1317-1335

12. Holmes RS, Goldberg E (2009) Computational analyses of mammalian lactate dehydrogenases human, mouse, opossum and platypus LDHs. Comput Biol Chem $33: 379-385$

13. Samland AK, Sprenger GA (2006) Microbial aldolases as C-C bonding enzymes unknown treasures and new developments. Appl Microbiol Biotechnol 71:253-264

14. Machajewski TD, Wong CH (2000) The Catalytic Asymmetric Aldol Reaction. Angew Chem Int Ed Engl 39:1352-1375

15. Anderson M, Scholtz JM, Schuster SM (1985) Rat liver 4-hydroxy-2ketoglutarate aldolase: purification and kinetic characterization. Arch Biochem Biophys 236:82-97

16. Kobes RD, Dekker EE (1969) 2-Keto-4-hydroxyglutarate aldolase of bovine liver. Purification, criteria of purity, and general properties. J Biol Chem 244:1919-1925

17. McLean A, Marwick MJ, Clayton BE (1973) Enzymes involved in phenylalanine metabolism in the human foetus and child. J Clin Pathol 26:678-683 
18. Alaux S, Kusk M, Sagot E, Bolte J, Jensen AA, Bräuner-Osborne H, Gefflaut T, Bunch L (2005) Chemoenzymatic synthesis of a series of 4-substituted glutamate analogues and pharmacological characterization at human glutamate transporters subtypes 1-3. J Med Chem 48:7980-7992

19. Maitra U, Dekker EE (1963) Enzymatic steps in the conversion of gammahydroxyglutamate to glyoxylate and alanine. J Biol Chem 238:3660-3669

20. Birdsey GM, Lewin J, Holbrook JD, Simpson VR, Cunningham AA, Danpure CJ (2005) A comparative analysis of the evolutionary relationship between diet and enzyme targeting in bats, marsupials and other mammals. Proc Biol Sci 272:833-840

21. Danpure CJ, Fryer P, Jennings PR, Allsop J, Griffiths S, Cunningham A (1994) Evolution of alanine:glyoxylate aminotransferase 1 peroxisomal and mitochondrial targeting. A survey of its subcellular distribution in the livers of various representatives of the classes Mammalia, Aves and Amphibia. Eur J Cell Biol 64:295-313

22. Danpure CJ (1993) Primary hyperoxaluria type 1 and peroxisome-tomitochondrion mistargeting of alanine:glyoxylate aminotransferase. Biochimie 75:309315

23. Coulter-Mackie MB, Lian Q, Wong SG (2005) Overexpression of human alanine:glyoxylate aminotransferase in Escherichia coli: renaturation from guanidine- $\mathrm{HCl}$ and affinity for pyridoxal phosphate co-factor. Protein Expr Purif 41:18-26

24. Hopper ED, Pittman AM, Fitzgerald MC, Tucker CL (2008) In vivo and in vitro examination of stability of primary hyperoxaluria-associated human alanine:glyoxylate aminotransferase. J Biol Chem 283:30493-30502

25. Caldwell EF, Mayor LR, Thomas MG, Danpure CJ (2004) Diet and the frequency of the alanine:glyoxylate aminotransferase Pro11Leu polymorphism in different human populations. Hum Genet 115:504-509 
26. Knight J, Easter LH, Neiberg R, Assimos DG, Holmes RP (2009) Increased protein intake on controlled oxalate diets does not increase urinary oxalate excretion. Urol Res 37:63-68 


\section{Figure legends}

Figure 1. Schematic representation of glyoxylate metabolic pathway in human hepatocytes; red circles/ellipses: enzymes that are mutant in primary hyperoxaluria; 4a: a cytosolic enzyme with 4-hydroxy-2-oxoglutarate aldolase activity.

Figure 2. Schematic representation of possible reactions with HOG and glyoxylate, catalyzed by cellular enzymes. See text for explanation of the steps.

Figure 3. Urinary concentrations of HOG and its derivatives measured by GC-MS. 2,4dihydroxyglutarate [1] and [2] represent meso-isomer and the mixture of two optically active isomers respectively. $\mathrm{X}$ axis - groups 1, 2 and 3 represent controls, obligatory carriers and PHIII patients respectively. $\mathrm{Y}$ axis $-\mu \mathrm{M} / \mathrm{mM}$ creatinine for $\mathrm{HOG}$ and 4-hydroxyglutamate and arbitrary units for 2,4-dihydroxyglutarate (logarithmic scale). The differences between groups 2 and 3, and groups 1 and 3 were statistically significant $(\mathrm{p}<0.01$, Mann-Whitney test).

Figure 4. 4-hydroxy-2-oxoglutarate aldolase activity in mitochondrial and cytosolic fractions of SK-HEP-1cells.

A: decrease of HOG concentrations following incubation with mitochondrial and cytosolic fractions of SK-HEP-1cells measured by GC-MS;

B: formation of glyoxylate and pyruvate from HOG in the cytosolic fraction measured in a coupled reaction with $\mathrm{LDH}$.

Figure 5. HOGA1 distribution in mitochondrial and cytosolic fractions of SK-HEP-1cells: Western with anti-HOGA1 antibodies; 1 - cytosolic fraction; 2 - mitochondrial fraction; 3 positive control (recombinant HOGA1); $\rightarrow$ Molecular weight standard.

Figure 6. Schematic representation of glyoxylate compartmentalization theory and mechanisms of cytosolic glyoxylate generation under pathologic conditions: A: normal conditions - no cytosolic glyoxylate; B: PHI: The total amount of glyoxylate or part of it 
(at AGT mistargeting - AGT*) is converted to glycolate by mitochondrial GRHPR and is recovered to glyoxylate in the peroxisomes which are lacking AGT activity. The accumulated glyoxylate exit into the cytosol. C: PHII: GRHPR deficiency leads to increased mitochondrial concentrations of glyoxylate and to its transport into the cytosol. D: PHIII: the accumulation of HOG in the mitochondria leads to its transport into the cytosol where it is converted to glyoxylate by a different cytosolic aldolase. Thick arrows - synthesis; thin arrows - transportation. 


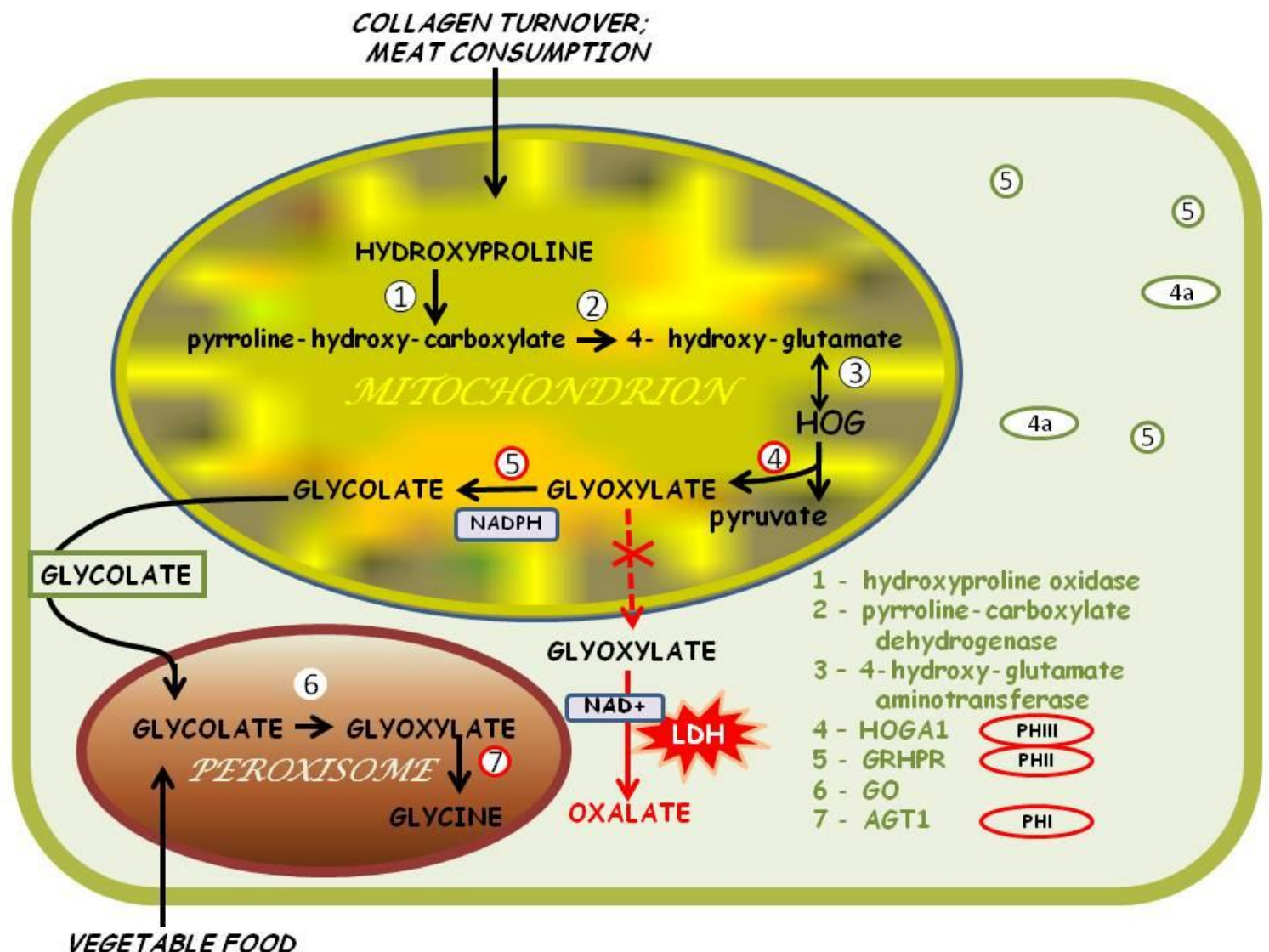




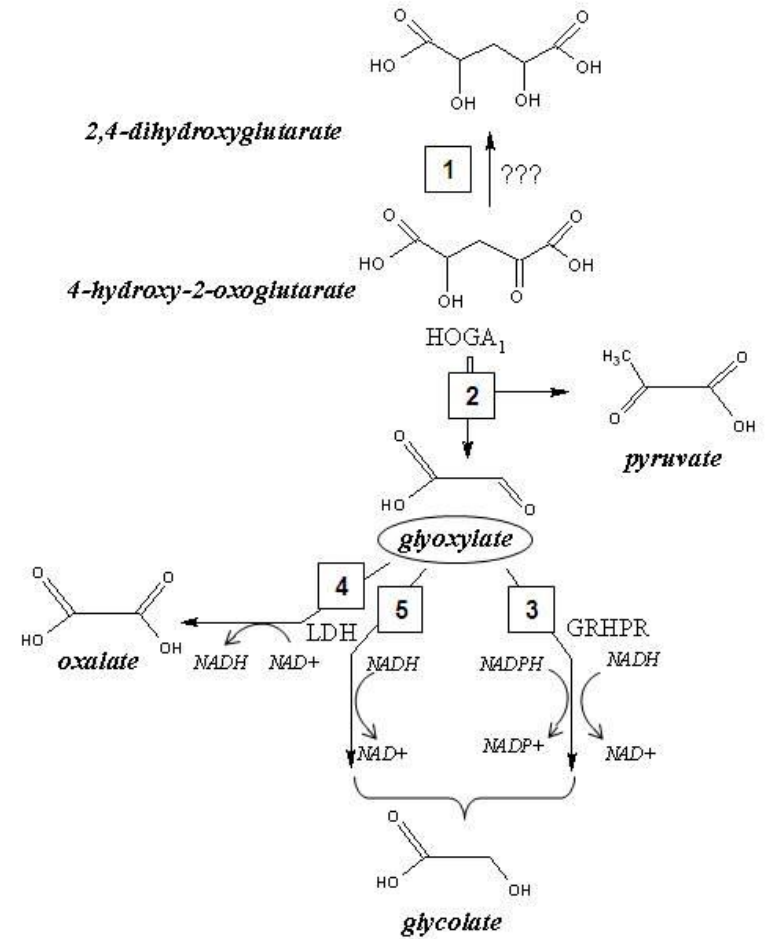



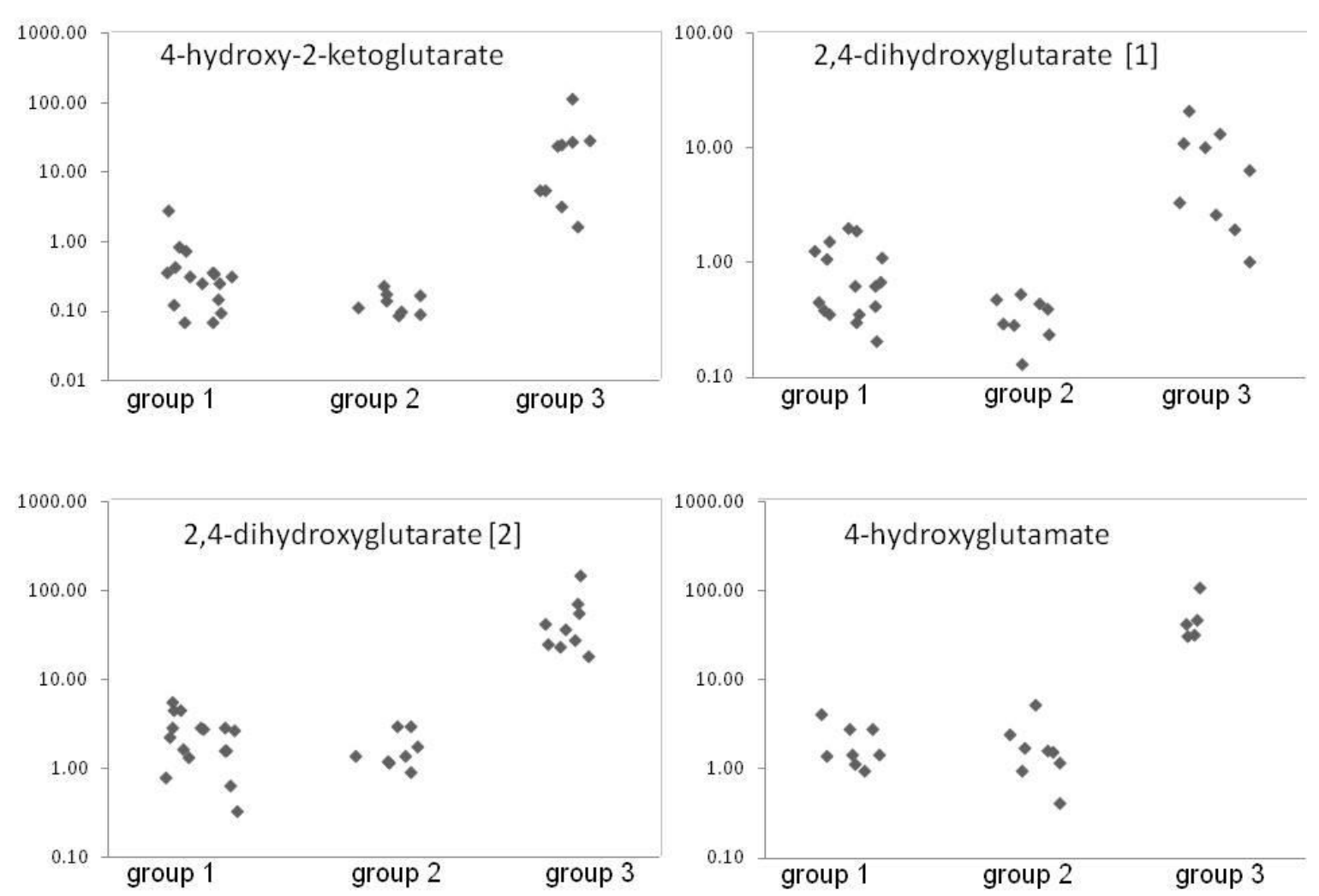

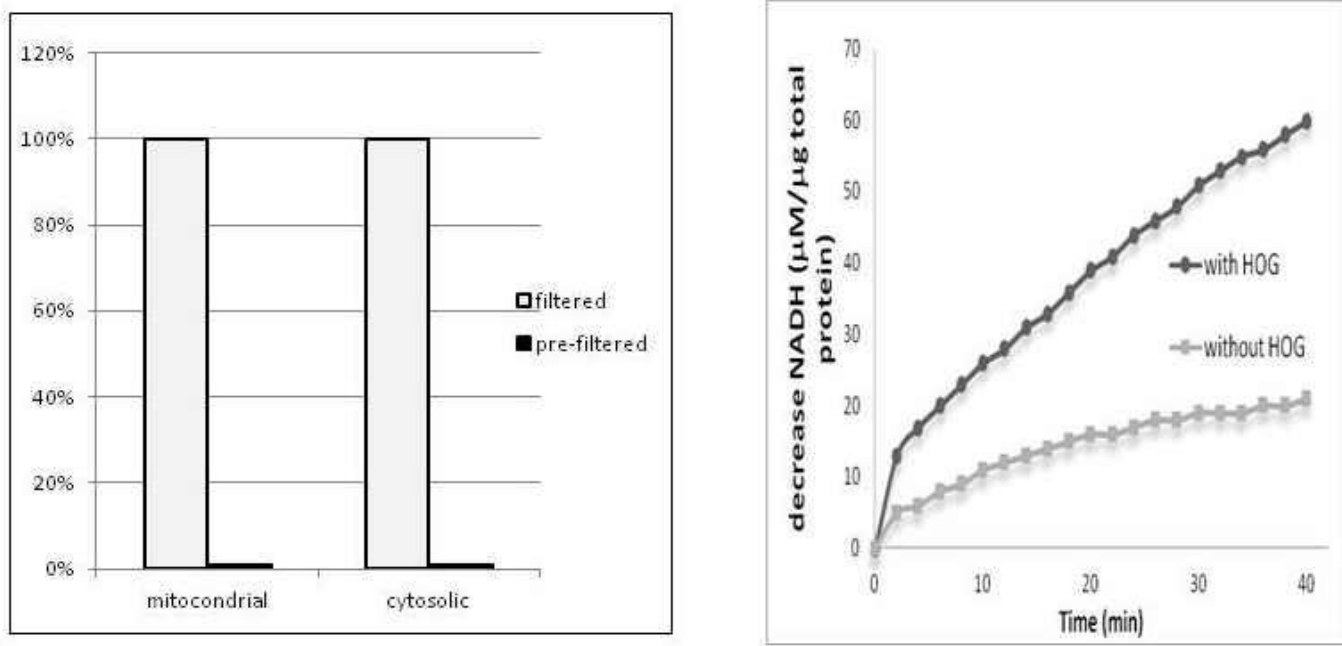


$$
123
$$

$36 \mathrm{kDa}$ 
MITOCHONDRION

A

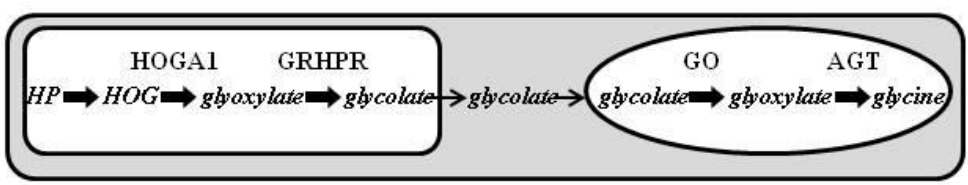

B

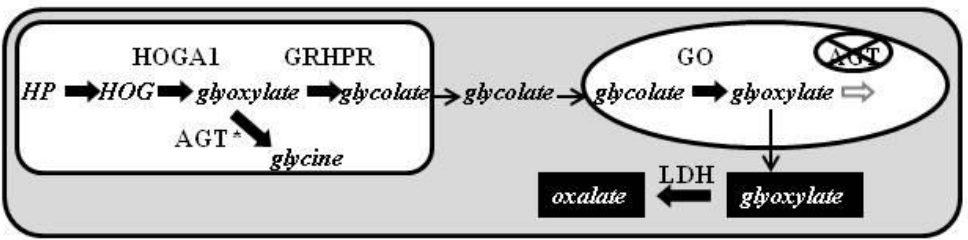

C

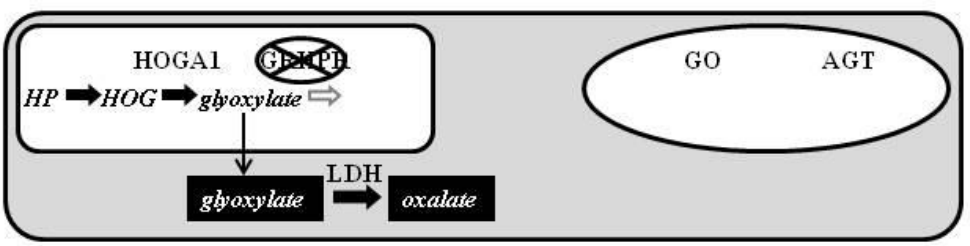

D

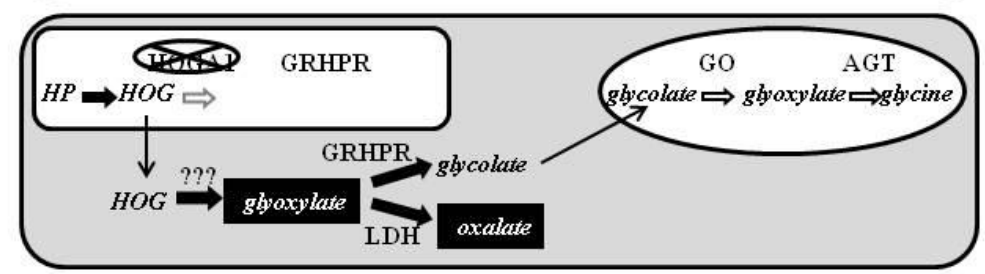

